\title{
THE PHASE DIAGRAM OF TWO-AXIS FERROMAGNET AND SPECTRA OF COUPLED MAGNETOELASTIC WAVES
}

\author{
Yu.N. Mitsay, Yu.A. Fridman, O.V. Kozhemyako \\ M.V. Frunze Simferopol State University \\ 333007, Yaltinskaya st. 4, Simferopol, Ukraine \\ AND M.S. KOCHMAŃSKI \\ Institute of Physics, University of Rzeszów \\ Rejtana 16A, 35-310 Rzeszów, Poland \\ (Received November 5, 1998; revised version March 22, 1999; \\ in final form July 13, 1999) \\ The phase diagram of strongly anisotropic two-axis ferromagnet at ar- \\ bitrary orientation of external magnetic field is built. It is demonstrated that \\ together with a ferromagnetic phase there is possible the existence of phases \\ with a tensorial order parameter: quadrupolar or quadrupolar-ferromagnetic \\ phases. Spectra of bound magnetoelastic waves are investigated. Besides it \\ is demonstrated that at large enough magnetoelastic interaction there is \\ possible an active interaction of a magnetic subsystem with longitudinally \\ polarized acoustic mode which becomes a soft mode at the point of orienta-
} tional phase transition.

PACS numbers: 75.10.-b

\section{Introduction}

It is well known that the account of magnetoelastic (ME) interaction results in a series of essentially dynamic effects in the vicinity of reorientation phase transitions (RPT), such as a softening of a spectrum of transversally polarized phonons and emerging of a ME gap in a magnon spectrum [1,2]. These effects are well enough investigated in the framework of phenomenological description $[1,2]$. However, such way of description based on the application of Landau-Lifshitz's equation and the equations of the theory of elasticity is limited to the condition of smallness of temperatures ( $T \ll T_{\mathrm{C}}, T_{\mathrm{C}}-$ Curie's temperature) and small values of one-ion anisotropy constants (OA). 
Recently, large interest has been evoked by the investigation of magnetics with more complex anisotropies than one-axis one, whose constants are comparable or even greater than a constant of exchange interaction. In such systems the realization of purely quantum effects is possible $[3,4]$, whose presence can essentially influence the dynamics of the system. The account of $M E$ interaction in such systems can result in a number of interesting effects. Thus, there is a problem of construction of the microscopic theory of bound ME waves which enables us to take exactly into account as large $\mathrm{OA}$ as $\mathrm{ME}$ interaction. It is possible to solve this problem using the Hubbard operators $[5,6]$.

\section{The dispersion equation of a two-axis ferromagnet}

As the system in study we shall consider a two-axis Heisenberg ferromagnetic, in an external magnetic field $\boldsymbol{H}$ parallel to the $O Z$. The Hamiltonian of such system can be written in the following form:

$$
\begin{aligned}
H= & -H \sum_{n} S_{n}^{z}+\frac{\beta_{1}}{2} \sum_{n}\left(S_{n}^{z}\right)^{2}+\frac{\beta_{2}}{2} \sum_{n}\left(S_{n}^{y}\right)^{2}+\frac{\beta_{3}}{2} \sum_{n}\left(S_{n}^{x}\right)^{2} \\
& -\frac{1}{2} \sum_{n, n^{\prime}} J\left(n-n^{\prime}\right) S_{n} S_{n^{\prime}}+\nu_{0} \sum_{n, i}\left(S_{n}^{i}\right)^{2} u_{i i}(n)+\nu_{1} \sum_{n, i, j} S_{n}^{i} S_{n}^{j} u_{i j}(n) \\
& +\int \mathrm{d} r\left(\frac{\lambda}{2} \sum_{i} u_{i i}^{2}+\mu \sum_{i, k} u_{i k}^{2}\right)
\end{aligned}
$$

where $\beta_{i}$ is the constant of OA; $J\left(n-n^{\prime}\right)>0$ is the exchange integral; $\nu_{i}$ is the constant of $\mathrm{ME}$ exchange interaction; $\lambda, \mu$ are the elasticity moduli.

We shall carry out calculations in terms of the Hubbard operators. For simplicity we shall take a magnetic ion spin $S=1$. The following scheme of calculations, however, is valid at $S>1$.

Separating in the exchange part of (1) mean fields $\left\langle S^{z}\right\rangle$, connected with the ordering of the magnetic moment, for a one-site Hamiltonian $H_{0}(n)$ we obtain

$$
\begin{aligned}
& H_{0}(n)=-\bar{H} S^{z}+\frac{\beta_{1}}{2}\left(S^{z}\right)^{2}+\frac{\beta_{3}-\beta_{2}}{8}\left[\left(S^{+}\right)^{2}+\left(S^{-}\right)^{2}\right] \\
& +\frac{\beta_{3}+\beta_{2}}{8}\left(S^{+} S^{-}+S^{-} S^{+}\right)+\frac{\nu_{0}}{4}\left\{\left[\left(S^{+}\right)^{2}+\left(S^{-}\right)^{2}\right]\left(u_{x x}-u_{y y}\right)\right. \\
& \left.+\left(S^{+} S^{-}+S^{-} S^{+}\right)\left(u_{x x}+u_{y y}\right)+4\left(S^{z}\right)^{2} u_{z z}\right\} \\
& \quad+\frac{\nu_{1}}{2}\left[\left(S^{+} S^{z}+S^{z} S^{+}\right) u_{z}^{+}+\left(S^{-} S^{z}+S^{z} S^{-}\right) u_{z}^{-}\right],
\end{aligned}
$$

where $\bar{H}=H+J(0)\left\langle S^{z}\right\rangle ; S^{+,-}=S^{x} \pm \mathrm{i} S^{y}, u_{z}^{+,-}=u_{x z} \pm \mathrm{i} u_{y z}$. Hamiltonian (2), expressed in terms of the Hubbard operators, built on eigenfunctions of the operator

$L=-\bar{H} S^{z}+\frac{\beta_{1}}{2}\left(S^{z}\right)^{2}+\frac{\beta_{3}-\beta_{2}}{8}\left[\left(S^{+}\right)^{2}+\left(S^{-}\right)^{2}\right]+\frac{\beta_{3}+\beta_{2}}{8}\left(S^{+} S^{-}+S^{-} S^{+}\right)$ 
has the form

$$
H_{0}(n)=\sum_{n}\left(\sum_{M} A_{M} H_{n}^{M}+\sum_{\alpha} A_{\alpha} H_{n}^{\alpha}\right) .
$$

Here $H_{n}^{M} \equiv X_{n}^{M, M}$ are diagonal Hubbard's operators, $M=-1,0,1, \alpha$ are corresponding root vectors. Solving Schrödinger's equation with Hamiltonian (3), we shall obtain the energy levels of a magnetic ion with the account of $M E$ interaction

$$
\begin{aligned}
& E_{1}=\frac{2 \beta_{1}+\beta_{2}+\beta_{3}}{4}+\frac{\nu_{0}}{2}\left(u_{x x}^{0}+u_{y y}^{0}+2 u_{z z}^{0}\right)-\frac{\chi}{2}, \\
& E_{0}=\frac{\beta_{2}+\beta_{3}}{2}+\nu_{0}\left(u_{x x}^{0}+u_{y y}^{0}\right), \\
& E_{-1}=\frac{2 \beta_{1}+\beta_{2}+\beta_{3}}{4}+\frac{\nu_{0}}{2}\left(u_{x x}^{0}+u_{y y}^{0}+2 u_{z z}^{0}\right)+\frac{\chi}{2},
\end{aligned}
$$

where

$$
\begin{aligned}
& \chi^{2}=\left[2 \chi_{0}-\nu_{0}\left(u_{x x}^{0}-u_{y y}^{0}\right) \sin 2 \theta\right]^{2}+\nu_{0}^{2}\left(u_{x x}^{0}-u_{y y}^{0}\right)^{2} \cos ^{2} 2 \theta, \\
& \chi_{0}^{2}=\bar{H}^{2}+\tilde{\beta}^{2}, \quad \tilde{\beta}=\frac{\beta_{3}-\beta_{2}}{4}, \quad \cos \theta=-\frac{\widetilde{\beta}}{\sqrt{2 \chi_{0}\left(\chi_{0}-\bar{H}\right)}} .
\end{aligned}
$$

The spontaneous deformations $u_{i j}^{0}$ are determined from the condition of a minimum of the free energy density $F=F_{0}-T \ln Z$, where $F_{0}$ is the density of elastic energy of the system, $Z=\sum_{M=1,0,-1} \exp \left(-E_{M} / T\right)$ is the partition sum.

The Hamiltonian $H_{0}$ is a nondiagonal one in the basis of eigenfunctions of the operator $L$. To diagonalize it we shall introduce the new Hubbard operators

$$
Y_{n}^{M^{\prime} M} \equiv\left|\widetilde{\Psi}_{n}\left(M^{\prime}\right)\right\rangle\left\langle\widetilde{\Psi}_{n}(M)\right| \quad\left(Y_{n}^{M} \equiv Y_{n}^{M M}\right),
$$

constructed on eigenfunctions $\widetilde{\Psi}_{n}(M)$ of Hamiltonian (3)

$$
\begin{aligned}
& \widetilde{\Psi}_{n}(1)=\cos \delta \Psi_{n}(1)+\sin \delta \Psi_{n}(-1), \quad \widetilde{\Psi}_{n}(0)=\Psi_{n}(0), \\
& \widetilde{\Psi}_{n}(-1)=\sin \delta \Psi_{n}(1)-\cos \delta \Psi_{n}(-1),
\end{aligned}
$$

where

$$
\cos \delta=\frac{\nu_{0}\left(u_{x x} u_{y y}\right) \cos 2 \theta}{\sqrt{2 \chi\left[\chi-2 \chi_{0}+2\left(u_{x x}-u_{y y}\right) \sin 2 \theta\right]}} .
$$

The relation of spin operators with the "new" Hubbard operators is as follows:

$$
\begin{aligned}
& S_{n}^{+}=\sqrt{2} \cos \tilde{\theta}\left(Y_{n}^{10}-Y_{n}^{0-1}\right)+\sqrt{2} \sin \tilde{\theta}\left(Y_{n}^{-10}+Y_{n}^{01}\right), \quad S^{-}=\left(S^{+}\right)^{+}, \\
& S_{n}^{z}=\cos 2 \tilde{\theta}\left(Y_{n}^{1}-Y_{n}^{-1}\right)+\sin 2 \theta\left(Y_{n}^{1-1}+Y_{n}^{-11}\right), \quad \tilde{\theta}=\theta-\delta .
\end{aligned}
$$

Representing components of tensor of deformations as $u_{i j}=u_{i j}^{0}+u_{i j}^{(1)}$, where $u_{i j}^{(1)}$ is the dynamic part of the tensor of deformations describing the oscillations of a crystal lattice and quantizing the dynamic part in a standard way [7], from Hamiltonian (3) we shall receive the Hamiltonian describing processes of transformation of magnon to phonon and vice versa

$$
H_{\mathrm{tr}}=\sum_{n}\left(\sum_{M} P_{M} Y_{n}^{M}+\sum_{\alpha} P_{\alpha} Y_{n}^{\alpha}\right)
$$


where

$$
P_{M(\alpha)}=\frac{1}{\sqrt{N}} \sum_{q, \lambda}\left(b_{q, \lambda}+b_{-q, \lambda}^{+}\right) T_{n}^{M(\alpha)}(q, \lambda)
$$

$b_{-q, \lambda}^{+}\left(b_{q, \lambda}\right)$ are rising (lowering) operators of phonons with polarization $\lambda$; $T_{n}^{M(\alpha)}(q, \lambda)$ are amplitudes of transformations.

The spectrum of elementary excitations is determined by poles of Green's function, which in our case looks like

$$
G^{\alpha \alpha^{\prime}}\left(n, \tau ; n^{\prime}, \tau^{\prime}\right)=-\left\langle T \tilde{Y}_{n}^{\alpha}(\tau) \tilde{Y}_{n^{\prime}}^{-\alpha^{\prime}}\left(\tau^{\prime}\right)\right\rangle .
$$

Here $T$ is a time-ordering operator, $\tilde{Y}_{n}^{\alpha}(\tau)$ is the Hubbard operator in the Heisenberg representation, and the averaging is made with the Hamiltonian $H=$ $H_{\text {int }}+H_{\text {tr }}$. We shall carry out all further calculations in a mean field approximation. The equation for Green's function is of Larkin's equation type

$$
\begin{gathered}
G^{\alpha \alpha^{\prime}}\left(k, \omega_{n}\right)=\Sigma^{\alpha \alpha^{\prime}}\left(k, \omega_{n}\right)-\frac{J(k)}{2} \Sigma^{\alpha \alpha_{1}}\left(k, \omega_{n}\right) A_{i}^{\alpha_{1}} B_{i}^{\alpha_{2}} G^{\alpha_{2} \alpha^{\prime}}\left(k, \omega_{n}\right) \\
+\Sigma^{\alpha \alpha_{1}}\left(k, \omega_{n}\right) T^{-\alpha_{1}}(k, \lambda) D_{\lambda}\left(k, \omega_{n}\right) T^{\alpha_{2}}(-k, \lambda) G^{\alpha_{2} \alpha^{\prime}}\left(k, \omega_{n}\right),
\end{gathered}
$$

where $\Sigma^{\alpha \alpha^{\prime}}\left(k, \omega_{n}\right)$ is nonreducible (by Larkin) part, $D_{\lambda}\left(k, \omega_{n}\right)=\frac{2 \omega_{\lambda}(k)}{\omega_{n}^{2}-\omega_{\lambda}^{2}(k)}$ is Green's function of a $\lambda$-polarized phonon with the dispersion law $\omega_{\lambda}(k) \stackrel{\omega_{\lambda}}{=} c_{\lambda} k$, $c_{\lambda}$ is the sound velocity, and columns $A_{i}^{\alpha}, B_{i}^{\alpha}$ have the form

$$
\begin{aligned}
& A_{1}^{\alpha}=\left(\begin{array}{c}
2 \gamma_{\|}(-\alpha) \\
\Gamma_{\|}(M)
\end{array}\right), \quad A_{2}^{\alpha}=\left(\begin{array}{c}
\gamma_{\perp}^{*}(\alpha) \\
0
\end{array}\right), \quad A_{3}^{\alpha}=\left(\begin{array}{c}
\gamma_{\perp}(-\alpha) \\
0
\end{array}\right), \\
& B_{1}^{\alpha}=\left(\begin{array}{c}
\gamma_{\|}(\alpha) \\
\Gamma_{\|}(M)
\end{array}\right), \quad B_{2}^{\alpha}=\left(\begin{array}{c}
\gamma_{\perp}(\alpha) \\
0
\end{array}\right), \quad B_{3}^{\alpha}=\left(\begin{array}{c}
\gamma_{\perp}^{*}(-\alpha) \\
0
\end{array}\right) .
\end{aligned}
$$

The functions $\gamma_{\perp(\|)}(\alpha)$ are determined by the relation of the spin operators with the Hubbard operators [6], and have the form (see (7)):

$$
\begin{aligned}
& \gamma_{\|}\left(\alpha_{1}\right)=\gamma_{\|}\left(\alpha_{2}\right)=-\sin 2 \tilde{\theta}, \quad \gamma_{\|}\left(\alpha_{i}\right)=0, \quad i=3,4,5,6, \\
& \gamma_{\perp}\left(\alpha_{1}\right)=\gamma_{\perp}\left(\alpha_{2}\right)=0, \quad \gamma_{\perp}\left(\alpha_{3}\right)=\gamma_{\perp}\left(\alpha_{5}\right)=\sqrt{2} \cos \tilde{\theta}, \\
& \gamma_{\perp}\left(\alpha_{4}\right)=-\gamma_{\perp}\left(\alpha_{6}\right)=\sqrt{2} \sin \tilde{\theta} .
\end{aligned}
$$

The root vectors $\alpha_{i}$ have the following components:

$$
\alpha_{1}=(1,0,-1)=-\alpha_{2}, \quad \alpha_{3}=(1,-1,0)=-\alpha_{4}, \quad \alpha_{5}=(0,1,-1)=-\alpha_{6} .
$$

Due to the split dependence of Eq. (8) on $\alpha$ it is possible to solve it, and taking into account that in the mean field approximation $\Sigma^{\alpha \alpha^{\prime}}\left(k, \omega_{n}\right)=\delta_{\alpha \alpha^{\prime}} G_{0}^{\alpha}\left(\omega_{n}\right) b(\alpha)$, where $G_{0}^{\alpha}\left(\omega_{n}\right)=\left[\mathrm{i} \omega_{n}+(\boldsymbol{\alpha} \boldsymbol{E})\right]^{-1}$ is zeroth Green's function, $b(\alpha)=\langle\boldsymbol{\alpha} \boldsymbol{E}\rangle$, we obtain the dispersion equation of bound ME waves

$$
\begin{aligned}
& \operatorname{det} \| \delta_{i j}+\frac{J(k)}{2} G_{0}^{\alpha}\left(\omega_{n}\right) b(\alpha) a_{i j}(\alpha) \\
& +\frac{J(k)}{2} B_{0} T^{-\alpha}(k, \lambda) G_{0}^{\alpha}\left(\omega_{n}\right) b(\alpha) T^{\beta}\left(-k, \lambda^{\prime}\right) G_{0}^{\beta}\left(\omega_{n}\right) b(\beta) a_{i j}(\alpha, \beta) \|=0 .
\end{aligned}
$$


In Eq. (9) we have introduced the following designations:

$$
\begin{aligned}
& B_{0}=\frac{D_{\lambda}\left(k, \omega_{n}\right)}{1-Q_{\lambda \lambda^{\prime}} D_{\lambda^{\prime}}\left(k, \omega_{n}\right)}, \\
& Q_{\lambda \lambda^{\prime}}=T^{\alpha}(-k, \lambda) G_{0}^{\alpha}\left(\omega_{n}\right) b(\alpha) T^{-\alpha}\left(k, \lambda^{\prime}\right), \\
& a_{i j}(\alpha, \beta)=\left(\begin{array}{ccc}
2 \gamma_{\|}(\alpha) \gamma_{\|}(-\beta) & \gamma_{\|}(\alpha) \gamma_{\perp}^{*}(\beta) & \gamma_{\|}(\alpha) \gamma_{\perp}(-\beta) \\
2 \gamma_{\perp}(\alpha) \gamma_{\|}(-\beta) & \gamma_{\perp}(\alpha) \gamma_{\perp}^{*}(\beta) & \gamma_{\perp}(\alpha) \gamma_{\perp}(-\beta) \\
2 \gamma_{\perp}^{*}(-\alpha) \gamma_{\|}(-\beta) & \gamma_{\perp}^{*}(-\alpha) \gamma_{\perp}^{*}(\beta) & \gamma_{\perp}^{*}(-\alpha) \gamma_{\perp}(-\beta)
\end{array}\right), \\
& b\left(\alpha_{1}\right)=\left[\exp \left(-\beta E_{1}\right)-\exp \left(-\beta E_{-1}\right)\right] / Z=-b\left(\alpha_{2}\right), \\
& b\left(\alpha_{3}\right)=\left[\exp \left(-\beta E_{1}\right)-\exp \left(-\beta E_{0}\right)\right] / Z=-b\left(\alpha_{4}\right), \\
& b\left(\alpha_{5}\right)=\left[\exp \left(-\beta E_{0}\right)-\exp \left(-\beta E_{-1}\right)\right] / Z=-b\left(\alpha_{6}\right),
\end{aligned}
$$

where $Z$ is the partition sum.

The dispersion equation (9) is valid at arbitrary temperatures up to Curie's temperature (excluding fluctuation area, which, naturally, is small enough), and also at any values of material constants $\left(\beta_{i}, a_{0}, J_{0}\right)$.

\section{Spectra of bound ME waves}

Let us analyse Eq. (9) for the most interesting case, when a wave vector $k \| O Y$. In such geometry the only nonzero components of a unit vector of polarization of phonon are $e_{l}^{y}, e_{\tau}^{x}, e_{t}^{z}$, and the dispersion equation (9) breaks down into two equations separately determining spectra of "longitudinal" and "transversal" oscillations, accordingly

$$
\left(1+x_{11}\right)\left|\begin{array}{cc}
1+x_{22} & x_{23} \\
x_{32} & 1+x_{33}
\end{array}\right|=0
$$

where

$$
x_{i j}=\frac{J(k)}{2}\left[G_{0}^{\alpha} b(\alpha) a_{i j}(\alpha)+B_{0} G_{0}^{\alpha} T^{-\alpha}(k) b(\alpha) G_{0}^{\beta} T^{\beta}(-k) b(\beta) a_{i j}(\alpha, \beta)\right] .
$$

The transformation amplitudes generally have cumbersome expressions, therefore we shall give only these of them, which will be necessary for us for the further calculations. In the given geometry we have:

for $\tau$-polarized phonons

$$
\begin{aligned}
& T^{\alpha_{1}}(k, \tau)=T^{\alpha_{2}}(k, \tau)=0, \\
& T^{\alpha_{3}}(k, \tau)=\frac{\nu_{1}}{2 \sqrt{2}} T_{0}(\cos \tilde{\theta}+\sin \tilde{\theta}) e_{\tau}^{z} k=-T^{\alpha_{4}}(k, \tau), \\
& T^{\alpha_{5}}(k, \tau)=-\frac{\nu_{1}}{2 \sqrt{2}} T_{0}(\cos \tilde{\theta}-\sin \tilde{\theta}) e_{\tau}^{z} k=-T^{\alpha_{6}}(k, \tau),
\end{aligned}
$$

for $l$-polarized phonons

$$
T^{\alpha_{1}}(k, l)=T^{\alpha_{2}}(k, l)=-\mathrm{i} \frac{\nu_{0}}{2} T_{0} \cos 2 \tilde{\theta} e_{l}^{y} k
$$


$T_{0}=\exp (\mathrm{i} k n) / \sqrt{2 m \omega_{\lambda}(k)}$. Such "splitting" of Eq. (9) is possible, since in our case $\gamma_{\perp}(\alpha) \gamma_{\|}(\beta)=0$ for all $\alpha, \beta$.

Let us consider further a low-temperature limit, i.e. $T \ll T_{\mathrm{C}}$. Besides we shall take into account that the ferromagnetic phase (FM), which the system in study is in, is stable in these two cases
1. $\beta_{3}>\beta_{1}>\beta_{2}$,
2. $\beta_{1}>\beta_{3}>\beta_{2}$.

These two cases actually correspond to the rotation of a magnetic field: in the first case the field is perpendicular to the easy axis and in the second - to the easy plane. Let us consider these two situations in detail.

Consider the first case, when the constants of OA satisfy the inequality $\beta_{3}>\beta_{1}>\beta_{2}$. In this case the magnetic field $H$ is parallel to the "mean" magnetization axis. Solving the dispersion equation (10), we shall obtain spectra of "longitudinal" and "transversal" branches of excitations, which in our geometry have the form

$$
\begin{aligned}
\varepsilon_{\|}(k) & =\sqrt{E_{1-1}\left[E_{1-1}+2 J(k) \sin ^{2} 2 \tilde{\theta}\right]}, \\
\omega_{\|}^{2}(k) & =\omega_{l}^{2}(k) \frac{E_{1-1}+2 J(k) \sin ^{2} 2 \tilde{\theta}+2 a_{0} \cos ^{2} 2 \tilde{\theta}}{E_{1-1}+2 J(k) \sin ^{2} 2 \tilde{\theta}}, \\
\varepsilon_{\perp}(k) & \stackrel{\leftrightarrow}{=} \sqrt{\left[E_{10}+J(k)(1+\sin 2 \tilde{\theta})\right]\left[E_{10}+J(k)(1-\sin 2 \tilde{\theta})\right]} \\
\omega_{\perp}^{2}(k) & =\omega_{\tau}^{2}(k) \frac{E_{10}+J(k)(1-\sin 2 \tilde{\theta})+a_{1}(1+\sin 2 \widetilde{\theta})}{E_{10}+J(k)(1-\sin 2 \tilde{\theta})},
\end{aligned}
$$

where $E_{i j}=E_{i}-E_{j}(i, j=1,0,-1) ; a_{0}=\nu_{0}^{2} / 2 \mu, a_{1}=\nu_{1}^{2} / 2 \mu, \omega_{l}(k)$ and $\omega_{\tau}(k)$ are spectra of longitudinally and transversally polarized non-interacting waves correspondingly.

Let us consider the case when the largest parameter of the system in study is the constant of exchange interaction $\left(J_{0} \gg \beta_{i}, a_{i}, H\right)$, i.e. the case of small OA. As we study the system at low temperatures, it is possible to account only the lowest energy level, which in this case is equal to $E_{1}$ and is determined by the expression (4).

In this case, the expression for $\left\langle S^{z}\right\rangle$ is

$$
\left\langle S^{z}\right\rangle=\cos 2 \tilde{\theta}=\sqrt{1-\left(\frac{\tilde{\beta}}{J_{0}}\right)^{2}} .
$$

and $\tau$-polarized sound wave interacts with the magnetic subsystem. The spectrum of quasiphonons, as follows from (13), assumes the form

$$
\omega^{2}(k)=\omega_{\tau}^{2}(k) \frac{\alpha k^{2}+H-H_{\mathrm{c}}}{\alpha k^{2}+H-H_{\mathrm{c}}+a_{1}},
$$

where $\alpha=J_{0} R_{0}^{2}, R_{0}$ is the radius of interaction, $H_{\mathrm{c}}=\frac{\beta_{1}-\beta_{2}}{2}$.

As follows from the expression (13) in a long-wave limit $\left(\alpha k^{2} \ll a_{1}\right)$ at $H=H_{\mathrm{c}}$ the quasiphonon branch of bound ME waves softens, i.e. its spectrum assumes the form

$$
\omega_{\perp}^{2}(k)=\omega_{\tau}^{2}(k) \frac{\alpha k^{2}}{a_{1}} .
$$


It testifies to the fact that at $H=H_{\mathrm{c}}$ the system undergoes orientational phase transition (OPT). With this in the spectrum of quasimagnons there appears the ME gap

$$
\varepsilon_{\perp}(0)=\sqrt{2 a_{1} \widetilde{\beta}}
$$

The longitudinally-polarized sound wave, as it follows from (11), practically does not interact with a magnetic subsystem and its spectrum is

$$
\omega_{\|}^{2}=\omega_{l}^{2}\left(1-\frac{a_{0}}{I_{0}}\right) \text {. }
$$

This situation corresponds to a well investigated case of weakly anisotropic easy axis ferromagnetic. Our results, in particular concerning the field OPT, are in good agreement with the results obtained with the use of the Landau-Lifshitz equation and the equations of the theory of elasticity.

It is of interest to investigate the behaviour of the system, when the constants of $\mathrm{OA}$ are comparable or even greater than the constant of exchange interaction. In this case (see, for example, $[3,4,8]$ ) the manifestation of quantum effects, such as "quantum reduction of spin" is possible.

However, the investigation of these effects is impossible on the basis of phenomenological equations, since it requires the precise account of $\mathrm{OA}$ and $\mathrm{ME}$ interaction.

We assume that $\beta_{3}>\beta_{1}>\beta_{2}$ and $\widetilde{\beta}>J_{0}, H$. In this case, as well as in the previous one, the lowest energy level is $E_{1}$. However, the mean magnetization, as follows from (7), will be smaller than the greatest possible value $\left\langle S^{z}\right\rangle=1$, and is determined by the expression $\left\langle S^{z}\right\rangle \approx H / \widetilde{\beta}$.

The reason of this reduction lies in the structure of the operators of OA- and ME-interaction in Hamiltonian (2). The operators link the state $|1\rangle$ and $|-1\rangle$, and the ground state of the system is a superposition of states $|1\rangle,|-1\rangle$. The greater the value of $\tilde{\beta}$, the greater the contribution of the state $|-1\rangle$, which results in the "quantum reduction" of $\left\langle S^{z}\right\rangle$.

As the analysis of spectra of bound ME waves shows, in this case there are no values of the magnetic field, at which the quasiphonon or quasimagnon branches soften. It means that the system does not undergo the OPT, and $\left\langle S^{z}\right\rangle$ is always parallel to the $O Z$ axis and decreases in absolute value in the process of reduction of the magnetic field $H$ value.

In our opinion, the interesting and not sufficiently enough investigated situation [9] is the case of large ME exchange constant. We still assume that $\beta_{3}>\beta_{1}>$ $\beta_{2}$. Besides $\widetilde{\beta}>J_{0}, H$, but $a_{0}>\widetilde{\beta}$. At the first sight such situation takes no place for real magnetoelastic systems. However, as it was shown in recent researches [9-11], in rare earths oxides (in particular, in vanadates $\mathrm{RVO}_{4}, \mathrm{R}=\mathrm{Pr}, \mathrm{Nd}, \mathrm{Tm}$ ) the parameter of $\mathrm{ME}$ exchange interaction is comparable or even greater than the exchange interaction. Besides, the nature of $\mathrm{OA}$ and $\mathrm{ME}$ is the same: spin-orbital interaction. Therefore it is reasonable to model such situation.

In this case the transversally polarized sound mode does not interact with the magnetic subsystem. However, a longitudinally polarized sound wave intensively interacts with a high-frequency magnon branch. 
The lowest energy level will be $E_{1}$, and the expression for $\left\langle S^{z}\right\rangle$ assumes the following form:

$$
\left\langle S^{z}\right\rangle \approx \frac{H}{\widetilde{\beta}+a_{0}}\left(1-\frac{J_{0}}{\widetilde{\beta}}\right) .
$$

It is in this case, as well as in the previous one, that the effect of "quantum reduction of spin" takes place, however, the parameter of ME exchange interaction plays the role of an "effective anisotropy" and essentially reduces $\left\langle S^{z}\right\rangle$. The reason of this reduction is the same as in the case of large OA.

As follows from (11), the spectrum of longitudinally polarized quasiphonons looks like

$$
\omega_{\|}^{2}(k)=\omega_{l}^{2}(k) \frac{\alpha k^{2}+H^{2}-H_{c 1}^{2}}{\alpha k^{2}+H^{2}-H_{\mathrm{c} 1}^{2}+a_{0}\langle S\rangle},
$$

while the spectrum of a high-frequency (relaxational) quasimagnon branch is

$$
\varepsilon_{\|}(k)=2 \sqrt{\alpha k^{2}+H^{2}+H_{c 1}^{2}+a_{0}\langle S\rangle\left(\tilde{\beta}+a_{0}\right)}
$$

In (16) and (17) the following designation is introduced:

$$
H_{\mathrm{c} 1}=2 \sqrt{\frac{2}{3} \tilde{\beta}\left(a_{0}-\frac{15}{8} \tilde{\beta}\right)} .
$$

As it is evident from (16) and (17), at $H=H_{\mathrm{c} 1}$ (in a long-wave limit $\left.\alpha k^{2} \ll a_{0}\langle S\rangle\right)$ the spectrum of longitudinally polarized quasiphonons softens

$$
\omega_{\|}^{2}(k)=\omega_{l}^{2}(k) \frac{\alpha k^{2}}{a_{0}\langle S\rangle_{c}}
$$

and in the spectrum of relaxational quasimagnons there appears a ME gap

$$
\varepsilon_{\|}(0)=2 \sqrt{a_{0}\langle S\rangle_{c}\left(\tilde{\beta}+a_{0}\right)}
$$

where $\langle S\rangle_{c}=\left\langle\left. S\right|_{H=H_{c 1}}\right.$.

It is necessary to note that such spectral dependence is possible, as it follows from (18), only at $a_{0}>1.75 \widetilde{\beta}$. If this is not so we come back to the earlier considered case of large $\mathrm{OA}$.

Thus, at $H=H_{\mathrm{c} 1}$ and $a_{0}>1.75 \tilde{\beta}>J_{0}$ the system undergoes OPT, connected with the rotation as well as with the reduction of the module $\left\langle S^{\prime}\right\rangle$, but under this conditions, the longitudinally polarized sound wave becomes a soft mode interacting with the relaxational magnon branch. We shall notice that for one-axis magnets the similar effect is not observed. It is connected with the structure of functions $\gamma_{\|}(\alpha)$, determining the relation between spin operators and Hubbard's operators, as well as with the structure of amplitudes of transformation.

Consider the spectra of bound ME waves in a strongly anisotropic two-axis $\mathrm{FM}\left(\beta_{i} \gg J_{0}\right)$, when the constants of OA justify $\beta_{1}>\beta_{3}>\beta_{2}$.

As it was shown in papers $[3,4,8]$, when the energy of OA is comparable with the exchange energy, the existence of new phases with a tensorial order parameter is possible. At fields $H_{\mathrm{c} 2}$ and $H_{\mathrm{c} 3}$ there occurs the OPT from the quadrupolar-under QU-phase characterized by the tensorial order parameter, to the quadrupolar-ferromagnetic (QFM) (angular), and from QFM to FM, in which 
the magnetic moment is directed along the field. Let us consider the spectra of bound ME waves in the following intervals of fields: $H<H_{\mathrm{c} 2}$ and $H>H_{\mathrm{c} 3}$.

At $H>H_{\mathrm{c} 3}$ the mean magnetization is directed along the field. The ground state in this case is $\widetilde{\Psi}_{n}(1)$, and the lowest energy level is $E_{1}$. The value of the mean magnetic moment is $\langle S\rangle \approx 1$.

The spectrum of quasiphonons in this phase is

$$
\omega_{\perp}^{2}(k)=\omega_{\tau}^{2}(k) \frac{\alpha k^{2}+H-H_{\mathrm{c} 3}}{a k^{2}+H-H_{\mathrm{c} 3}+a_{1}},
$$

and in a long-wave limit at $H=H_{\mathrm{c} 3}=\beta=\left(2 \beta_{1}-\beta_{3}-\beta_{2}\right) / 4$ it softens. In the spectrum of quasimagnons there appears the ME gap (at $H=H_{\mathrm{c} 3}$ ): $\varepsilon_{\perp}(0)=$ $\sqrt{2 a_{1} J_{0} \beta / \widetilde{\beta}}$

Let us consider now the spectrum of ME waves at $H<H_{\mathrm{c} 2}$. In this case there takes place an inversion of energy levels, and as follows from (4), the lowest level of a magnetic ion is $E_{0}$. As follows from (7) the magnetic order parameter $\left\langle S^{z}\right\rangle=0$, but the tensorial order parameter $3\left\langle\left(S^{z}\right)^{2}\right\rangle-2 \neq 0$. Thus, the system is in a $Q U$-phase with a ground state $\widetilde{\Psi}(0)$. In this case the mechanism of realization of a. QU-phase differs from the corresponding mechanism at "quantum reduction of spin". It is connected with the difference of corresponding ground states.

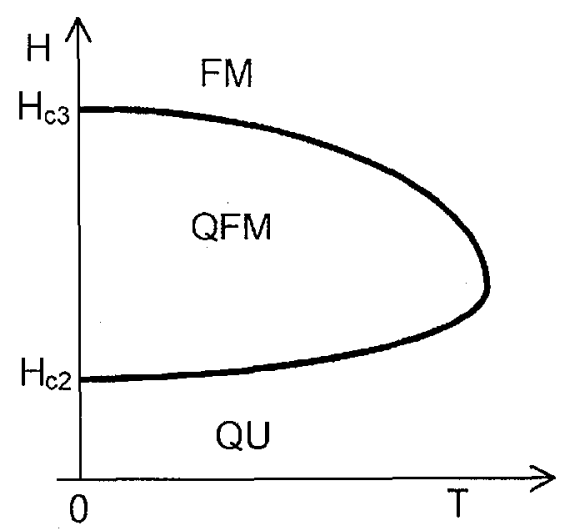

Fig. 1. The phase diagram of a strongly anisotropic two-axis ferromagnet at $H \| O Z$.

From Eq. (9) it follows that $l$ - and $t$-polarized sound modes do not interact with the magnetic subsystem, and for the spectrum of $\tau$-polarized quasiphonons we obtain

$$
\omega_{\perp}^{2}(k)=\omega_{\tau}^{2}(k) \frac{\alpha k^{2}+H_{c 2}^{2}-H^{2}}{\alpha k^{2}+H_{c 2}^{2}-H^{2}+\widetilde{a}_{1}},
$$

where $\tilde{a}_{1}=2 a_{1}\left(\beta-\tilde{\beta}-2 I_{0}\right), H_{\mathrm{c} 2}=\sqrt{\left(\beta+\widetilde{\beta}-2 a_{1}\right)\left(\beta-\widetilde{\beta}-2 I_{0}\right)}$. At $H=H_{\mathrm{c} 2}$ in the spectrum of quasimagnons there appears a $\mathrm{ME}$ gap

$$
\varepsilon_{\perp}(0)=\sqrt{2 a_{\perp} J_{0} \widetilde{\beta}(\beta-\widetilde{\beta})} / \beta \text {. }
$$

The phase diagram of the system for the described situation (in variables $(H, T))$ is given in Fig. 1. Similar phase diagrams for one-axis ferromagnets with 
large $\mathrm{OA}$ were obtained in $[3,4]$. As is obvious from the phase diagram, at $H=H_{\mathrm{c} 3}$ the system undergoes the phase transition from FM to angular (QFM) phase, and at $H=H_{\mathrm{c} 2}$, from QU-phase to QFM.

\section{The phase diagram of a two-axis ferromagnet}

Let us consider now the case when the magnetic field is arbitrarily directed in the plane $Z O X$, and obtain the phase diagram of the described system in such geometry.

Let us assume that the magnetic field lies in the plane $Z O X$ at an angle $\alpha$ to the $O Z$ axis. The limiting cases of such geometry were considered previously $(\alpha=0, \alpha=\pi / 2)$. We also assume that OA is great $\left(\tilde{\beta}>J_{0}\right)$. As earlier, we shall consider the case of low temperatures.

Let us rotate the system of coordinates around the $O Y$ axis by an angle $\alpha$ so that the new axis of quantization $O Z^{\prime}\left(O Z^{\prime} \| \boldsymbol{H}\right)$ becomes parallel to the vector of magnetization. In this local system of coordinates we introduce new spin operators $\widetilde{S}_{n}^{x}, \widetilde{S}_{n}^{y}, \widetilde{S}_{n}^{z}$, in terms of which the one-site Hamiltonian has the form

$$
\begin{aligned}
& H_{0}(n)=-\bar{H} \widetilde{S}^{z}+\frac{B_{1}}{2}\left(\widetilde{S}^{z}\right)^{2}+\frac{\beta_{2}}{2}\left(\widetilde{S}^{y}\right)^{2}+\frac{B_{3}}{2}\left(\widetilde{S}^{x}\right)^{2} \\
& +\frac{\beta_{3}-\beta_{1}}{4} \sin 2 \alpha\left(\widetilde{S}^{z} \widetilde{S}^{x}+\widetilde{S}^{x} \widetilde{S}^{z}\right),
\end{aligned}
$$

where $B_{1}=\beta_{1} \cos ^{2} \alpha+\beta_{3} \sin ^{2} \alpha, B_{3}=\beta_{1} \sin ^{2} \alpha+\beta_{3} \cos ^{2} \alpha$.

Expression (20) formally differs from the one-site Hamiltonian (2) by the presence of the last term, proportional to $\sin 2 \alpha$. Therefore if we restrict ourself to the cases $\alpha \rightarrow 0$ and $\alpha \rightarrow \pi / 2$, we come back to an earlier examined situation (see Sec. 3), with formal replacement $\beta_{1} \rightarrow B_{1}$ and $\beta_{3} \rightarrow B_{3}$. The phase diagram of the system in this case (in variables $\left(H_{x}, H_{z}\right)$ ) is given in Fig. 2. Line 1 separates FM- and QFM-phase. This line passes through the point $H_{\mathrm{c} 3}$, through the point $H_{1 x}=\frac{4}{9}\left(H_{\mathrm{c} 3}\right)^{3 / 2}$, the tangent to this line in the point $O$ is determined by the equation $\cos 2 \alpha=-J_{0} / \beta^{\prime}$, where $\beta^{\prime}=\frac{\beta_{1}-\beta_{3}}{4}$. In the case $\cos 2 \alpha<-J_{0} / \beta^{\prime}$, the system is in a FM-phase.

Line 2 separates QFM- from a QU-phase. This line passes through points $H_{\mathrm{c} 2}$, and

$$
H_{2 x}=\sin \alpha \sqrt{\left(\frac{\beta_{1} \cos ^{2} \alpha+\beta_{3} \sin ^{2} \alpha-\beta_{2}}{4}-2 a_{1}\right)\left(2 \beta^{\prime} \cos 2 \alpha-2 J_{0}\right)}
$$

$\left(\sin ^{2} \alpha=\frac{1}{4}\left(1-2 \frac{\beta^{\prime}}{\beta_{1}-\beta_{2}}\right)\right)$, and the equation of the tangent to line 2 in point $O$ has the form $\cos 2 \alpha=J_{0} / \beta^{\prime}$.

If an angle of the direction of a magnetic field with the axis $O Z$ satisfies inequality $-J_{0} / \beta^{\prime}<\cos 2 \alpha<J_{0} / \beta^{\prime}$, the system can exist both in FM- and in QFM-phase, depending on the value of an external magnetic field.

If $\cos \alpha>J_{0} / \beta^{\prime}$, the system can exist in any of these three phases (FM, $\mathrm{QFM}$, and $\mathrm{QU}$ ) depending on the value of a field. The obtained phase diagram describes well the limiting cases $\alpha=0, \alpha=\pi / 2$, investigated in detail in Sec. 3 . It is necessary to note that on lines 1 and 2 (Fig. 2) the system undergoes phase 


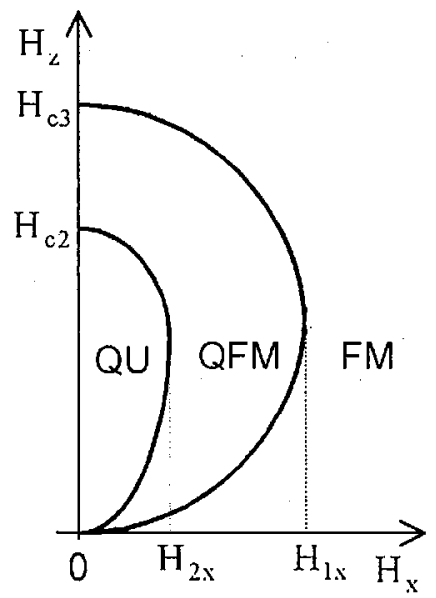

Fig. 2. The phase diagram of a strongly anisotropic two-axis ferromagnet in an external field, arbitrarily directed in the plane $Z O X$.

transitions of the second type, while the unstable ("soft") mode is a transversally polarized quasiphonon branch, and in a quasimagnon branch of excitation there appears a ME gap.

\section{Conclusion}

The researches carried out show that two-axis FMs have a number of specific features in comparison with one-axis ones. A special interest represents the case of strongly anisotropic magnets. In this case, as was shown above, the realization of a phase with a tensorial order parameter is possible. Depending on a relation between constants of $O A$, this phase can be realized in different ways. The phase diagram of such systems has no analogues for one-axis magnets.

In the case of large ME exchange, $t$ - and $\tau$-polarized acoustic modes do not interact with a magnetic subsystem and $l$-polarized acoustic modes actively interact with a relaxational quasimagnon branch. At critical values of the field, the longitudinally polarized acoustic modes become soft modes, i.e. the OPT is realized, at which the length of a magnetization vector also changes.

Let us note that in the experiment [12] there was revealed the striking correlation of the high-frequency characteristics and the acoustic ones in a magnetic transition in $\mathrm{ErFeO}_{3}$, caused by longitudinal oscillations of magnetization.

Such behaviour of $l$-polarized acoustic wave is not observed in one-axis FM or $\mathrm{FM}$ with small $\mathrm{OA}$ and small value of a $\mathrm{ME}$ parameter. Hence, it is possible to assert that, first, this effect is connected with the symmetry of a crystal, and second, with the effect of "quantum reduction of spin".

\section{References}

[1] E.A. Turov, V.G. Shaviov, Usp. Fiz. Nauk 140, 429 (1983).

[2] V.I. Ozhogin, V.L. Preobrazhenskii, Usp. Fiz. Nauk 155, 593 (1977).

[3] Yu.N. Mitsay, Yu.A. Fridman, Fiz. Tverd. Tela 32, 361 (1990). 
[4] F.P. Onufrieva, Fiz. Tverd. Tela 26, 3435 (1984).

[5] R.O. Zaitsev, Zh. Eksp. Teor. Fiz. 68, 207 (1975).

[6] Yu.N. Mitsay, Yu.A. Fridman, Teor. Mat. Fiz. 89, 207 (1989).

[7] L.D. Landau, E.M. Lifshits, Statistical Physics, Part 1, Nauka, Moskva 1976, p. 583.

[8] V.M. Loktev, V.S. Ostrovskii, Fiz. Nizk. Temp. 20, 983 (1994).

[9] V.D. Buchelnikov, V.G. Shavrov, Fiz. Tverd. Tela 37, 1402 (1995).

[10] Z.A. Kazei, N.P. Kolmakova, Zh. Eksp. Teor. Fiz. 109, 1687 (1996).

[11] V.D. Buchelnikov, N.K. Danshin, L.T. Tsimbal, V.G. Shavrov, Usp. Fiz. Nauk 166, 585 (1996).

[12] N.K. Danshin, L.T. Tsimbal, Zh. Eksp. Teor. Fiz. 106, 1765 (1994). 